\title{
Intelligent Learning and Control of Autonomous Robotic Agents Operating in Unstructured Environments
}

\author{
Hani Hagras*, Tarek Sobh** \\ *Department of Computer Science, University of Essex, Wivenhoe Park, Colchester \\ CO4 3SQ, England, UK
}

\author{
**School of Engineering and Design, University of Bridgeport, 169 University \\ Avenue, Bridgeport, Connecticut 06601, U.S.A.
}

\begin{abstract}
The control of autonomous intelligent robotic agent operating in unstructured changing environments includes many objective difficulties. One major difficulty concerns the characteristics of the environment that the agent should operate in. In unstructured and changing environments the inconsistency of the terrain, the irregularity of the product and the open nature of the working environment result in complex problems of identification, sensing and control. Problems can range from the effects of varying environmental conditions on the robot sensors and traction performance through to the need to deal with the presence of unexpected situations. Another major challenge is the large amounts of uncertainty that characterises real-world environments. On the one hand, it is not possible to have exact and complete prior knowledge of these environments. On the other hand, knowledge acquired through sensing is affected by uncertainty and imprecision. The quality of sensor information is influenced by sensor noise, the limited field of view, the conditions of observation, and the inherent difficulty of the perceptual interpretation process. Because environments and users of systems continuously change, robotic agents have to be adaptive. Intelligence helps because it gives systems the capacity to adapt more rapidly to environmental changes or to handle much more complex functions. In his paper we introduce this special issue and introduce the difficulty robots are facing in unstructured environments and how learning and computational intelligence can help the robots to adapt and give them the necessary intelligence they to face the challenges they encounter in their environments.
\end{abstract}

\section{Introduction}

Autonomous Robotic agents are aimed to build physical systems that can accomplish useful tasks without human intervention operating in unmodified real-world environments, i.e. in environments that have not been specifically engineered for the agent. To accomplish a given task, an agent collects or receives sensory information concerning its external environment and takes actions within the dynamically changing environment. However, the control rules are often dictated by human operators, although ideally the agent should automatically perform the tasks without assistance. Consequently the agent must be able to perceive the environment, make decisions, represent sensed data, acquire knowledge, and infer rules concerning the environment. Agents that can acquire and usefully apply knowledge or skill are often called Intelligent, these agents receive a task from a human operator and must accomplish the task in the available workspace [7]. 
The control of autonomous intelligent mobile robotic agent operating in unstructured changing environments includes many objective difficulties. One major difficulty concerns the characteristics of the environment. In outdoor environments, such as the agricultural environment and under water environments the inconsistency of the terrain, the irregularity of the product and the open nature of the working environment result in complex problems of identification, sensing and control. Such problems can range from the effects of varying weather conditions on the robot sensors and traction performance, through to the need to deal with the presence of unauthorised people. Another major challenge for autonomous mobile robotic agents operating in changing unstructured environments is the large amounts of uncertainty that characterises these real-world environments. On the one hand, it is not possible to have exact and complete prior knowledge of these environments: many details are usually unknown, the position of people and objects cannot be predicted a priori, passageways may be blocked, and so on. On the other hand, knowledge acquired through sensing is affected by uncertainty and imprecision. Sensor noise, the limited field of view, the conditions of observation, and the inherent difficulty of the perceptual interpretation process can all influence the quality of the sensor information.

Online learning and adaptation and life long learning using real robots are desirable traits for any robot learning algorithm operating in changing and unstructured environments where the robot explores its environment to collect sufficient samples of the necessary experience. Online learning is useful for producing intelligent machines for inaccessible environments such as underwater, flying robots (helicopters) or where reprogramming the robots would be difficult or expensive. In these environments it is required to perform online learning through interaction with the real environment and performing any adaptation within short time intervals.

\section{Intelligent Autonomous Embedded Agents}

According to Steels [24] an agent is a system that is capable of maintaining itself. An agent therefore must worry about two things: i) performing the function (or set of functions) that determines its role and ii) maintaining its own viability. This definition of an agent is so far almost completely identical with that of a living system as living systems must continuously replace their components to secure their existence in the face of unreliable or short lived components. These systems must also adapt or evolve to remain viable even if their environment changes.

The central idea in the concept of autonomy is identified in the etymology of the term: autos (self) and nomos (rule or law). It was first applied to the Greek city states whose citizens made their own laws, as opposed to living according to those of an external governing power [24]. To be autonomous you must first be automatic. This means that you must be able to operate in an environment, sense this environment and impact it in ways that are beneficial to yourself and to those tasks that are crucial to your further existence. But autonomy goes beyond automaticity, because it also supposes that the basis of self-steering originates (at least partly) in the agent's own capacity to form and adapt its principles of behaviour. Moreover the process of building up or updating competence is something that takes place, while the agent is operating in the real environment (not in simulation). It is not the case that the agent has the time to study a large number of examples or to think deeply about how it could cope with unforeseen circumstances. Instead, it must continuously act and respond in order to survive. AI systems built using the classical approach are not autonomous, although they are automatic. Knowledge has been extracted from experts and put into the system explicitly. It is not done by the system itself. Current robotic systems are also automatic but so far not autonomous. These systems can never step outside the boundaries of what was foreseen by the designers because they cannot change their own behaviour in a fundamental way. Biological systems are autonomous. Their structure is not built up by an outside agency, but they develop and maintain their internal structure and functioning through mechanisms like 
self-organisation, evolution, adaptation, and learning; and they do so while remaining viable in the environments in which they operate [24].

According to Kasabov [15] an Intelligent Agent System (IAS) should be able to learn quickly from large amounts of data. He also states that an intelligent system should be able to adapt in real time and in an on-line mode as new data is encountered. The system should also be able to accommodate, in an incremental way, any new problem solving rules as they become known. It should be memory-based, plus possess data and exemplar storage and retrieval capacities. In addition, he says that an IAS should be able to learn and improve through active interaction with the user and the environment. It should have parameters to represent short and long term memory, age, forgetting, etc. Finally he states it should be able to analyse itself in terms of behaviour, error and success.

In summary we can say that robotic agents are self-sustaining systems which perform a function for others and thus get the resources to maintain themselves. But because they have to worry about their own survival they need to be autonomous, both in the sense of self-governing and of having their own motivations. Because environments and users of systems continuously change, robotic agents have to be adaptive. Intelligence helps because it gives systems the capacity to adapt more rapidly to environmental changes or to handle much more complex functions.

\section{Online Learning versus Simulation}

A first fundamental requirement for robotic agents is that agents must be grounded in that they must be able to carry on their activities in the real world, in real time according to the above definition of robotic agents. Another important point is that adaptive behaviour cannot be considered as a product of an agent considered in isolation from the world, but can only emerge from strong coupling of the agent and its environment. Despite this, many embedded agent researchers regularly use simulations to test their models. However, the validity of such computer simulations to build autonomous robotic agents is often criticised and the subject of much debate. Even so computer simulations may still be very helpful in the training and testing of agents models. However as Brooks [4] pointed out "it is very hard to simulate the actual dynamics of the real world". This may imply that effort will go into solving problems that simply do not come up in real world with a physical robot and that programs which work well on simulated robots will completely fail on real robots. There are several reasons why those using computer models (simulations) to develop control systems for embedded agents operating in unstructured and changing environments may encounter problems [21]:

a) Numerical simulations do not usually consider all the physical laws of the interaction of a real agent with its own environment, such as mass, weight, friction, inertia, etc.

b) Physical sensors deliver uncertain values, and commands to actuators have uncertain effects, whereas simulative models often use grid-worlds and sensors that return perfect information.

c) Physical sensors and actuators, even if apparently identical, may perform differently because of slight variations in the electronics and mechanics or because of their different positions on the robot or because of the changing weather or environmental conditions.

Evolving the robot controllers online enables the learnt controller to adjust to the real noise and imprecision associated with the sensors and actuators. By doing this we can develop rules that take such defects into account, producing a realistic controller for autonomous robotic agents, grounded in the physical world that emerge from strong coupling of the agent and its environment not in simulation. These agents are grounded in the real world (situated, embodied and operating in real time), as adaptive behaviours cannot be considered as a product of an agent in isolation from the world, but can only emerge from strong coupling of the agent and its environment. 


\section{Methods used to develop robotic agents}

\subsection{Path Planning}

The navigation of a robotic agents can be considered as a task of determining a collision free path that enables the vehicle to travel through an obstacle course from an initial configuration to a goal configuration. The process of finding such path is also known as the path planning problem which could be classified into: global path planning and local path planning.

Global path planning methods are usually conducted off-line in a completely known environment [27]. In the global path planning approach, an exact model of the environment has to be used to plan the path. In addition, the time complexity grows exponentially with the geometry complexity and the number of degrees of freedom in the vehicle's motion. Thus they are only practical when the environmental model is simple and the number of degrees of freedom is reasonably low. However real environments are never simple enough. On the other hand local path planning techniques, also known as the obstacle avoidance methods, are potentially more efficient in robot navigation when the environment is unknown (which is our case) or only partially known [27].

An efficient local path planning method is the potential field method, which was first proposed by Khatib [17] and has been widely used in obstacle avoidance [3]. In spite of its simplicity and elegance, this method has three problems. First, local minimum could occur and cause the vehicle to be stuck. Second it can cause unstable motion in the presence of obstacles. Finally, it is difficult to find the force coefficients influencing the vehicle's velocity and direction in unknown environments [27]. The practicality of the potential field therefore hinges on how well these problems can be resolved. One of the possible solutions that has the potential to overcome these problems is the reactive system proposed by Brooks [4]. The key idea is to build a mapping from the perceived situations to the correct actions and iterate the mapping until a goal is reached. As previous reactive systems are not able to acquire situation-action rules, neural networks and fuzzy logic approaches offer an attractive alternative for building reactive systems in recent times [25].

\subsection{Neural Networks}

Neural networks had been used for embedded agents development namely the navigation of robots [22]. Neural Networks have the ability to learn by example and generalise their behaviour to new data, however these supervised learning methods require substantially large sets of representative patterns to characterise the environment during training. Also it is difficult to obtain the training patterns which contain no contradictory input output pairs. Further, it is well known that these networks learn rather slowly in high dimensional spaces (which includes robots operating in unstructured environments). In addition the neural network structure, i.e. the number of hidden units, needs to be chosen carefully to achieve good learning results. Too many nodes in the hidden layers of the network may cause the network to overfit the training data, while too few may reduce its ability to generalise. Selection of the optimal number of layers and nodes is a difficult problem that is widely solved by a 'rule of thumb' approach, which is directly proportional to the experience of the neural network designer [16]. Since an autonomous mobile robotic agent in a changing unstructured environment will encounter new data all the time, complex off-line retraining procedures would need to be devised and substantial amount of data would have to be stored for the retraining [26]. 


\subsection{Fuzzy Logic}

Fuzzy sets and systems constitute one of the most fundamental and influential computational intelligence tools [25]. Given the uncertain and incomplete information an autonomous robotic agent has about the environment, fuzzy rules provide an attractive means for mapping sensor data to appropriate control actions in real time. The success of Fuzzy Logic Control (FLC) is owed in a large part to the technology's ability to convert qualitative linguistic descriptions into complex mathematical functions and the ability to deal with various situations without analytical model of the environment. The methodology of the FLC appears very useful when the processes are too complex for analysis by conventional quantitative techniques or when the available sources of information are interpreted qualitatively, inexactly or uncertainly. Which is the case of autonomous robots navigating in unstructured environments. The fuzzy logic approach seems quite promising in tackling the problem of obstacle avoidance, as it deals with various situations without requiring to construct an analytical model of the environment. When compared with the neural network approach, it has another distinct advantage in that each rule of the rule base has a physical meaning. This makes it possible to tune the rules by using expert knowledge. However in complex unstructured environments the necessity of a high quality information, to be extracted from a broad knowledge domain, constrains the application of fuzzy systems to solve very demanding problems. Even if a human expert can help in the specification of such a complex system, improper solutions maybe produced, since there is a high probability of neglecting some important aspects and overemphasising others. Also as the number of inputs variables increases (which is the case of embedded agents) the number of rules increase exponentially which creates much difficulty in determining large numbers of rules. In the case of navigating the mobile vehicle in complex environments, it is difficult to consistently construct the rules since there are many situations to be handled; and it is time consuming to tune the constructed rules using human experience [27]. That is why automatic design of fuzzy systems represents a very promising and challenging research area [5] where our techniques will be used.

\subsection{Evolutionary algorithms}

Evolutionary algorithms constitute a class of search and optimization methods guided by the principles of natural evolution and genetics. It is the case that Genetic Algorithms (GA) have been successfully applied to solve a variety of difficult theoretical and practical problems by imitating the underlying processes of evolution such as selection, recombination and mutation. GA posses the following advantages, they are efficient, possessing the ability to solve certain combinatorial problems many orders of magnitude faster than iterative searches. They are problem independent not based on gradient information and therefore has no requirement on continuity or convexity of the solution space, caring nothing of the problem being solved, asking only that the solution be rated according how well it solves the problem. They are implicitly parallel where the solution is explored in parallel by searching different regions, this characteristic allows a global search in the solution space [16]. The GA approach enriches the optimization environment for fuzzy systems. As described in [5], developing an optimal fuzzy system is equivalent to finding the minimum of a hyper-surface associated with an objective function. The hyper-surface has the following characteristics: It is infinitely large, complex and noisy; it is non-differentiable, since changes in the number of fuzzy rules are discrete and can have a discontinuous effect on the fuzzy system's performance. It is multi-modal (different fuzzy rule sets and/or membership functions may have similar performance) and deceptive, since a little modification may cause huge effects on the performance of each system [5]. There is much work reported in the literature on designing fuzzy controllers using GA $[2,7,6,7,13,18,19,20,21]$. However virtually most of this work was 
undertaken using simulation as in conventional GA, it takes a large number of iterations to develop a good controller. Thus it is not feasible for a simple GA to learn online and adapt in real-time. The situation is worsened by the fact that most evolutionary computation methods developed so far assume that the solution space is fixed. That is, the evolution takes place within a pre-defined problem space and not in a dynamically changing and open one, thus preventing them from being used in real-time applications [15].

Hagras et al $[8,9,10,11,12]$ had produced a double hierarchical fuzzy genetic system for online learning and adaptation of mobile robots in outdoor and unstructured environments. In their system the online learning mechanism, in addition to the fuzzy behaviours are organised as a hierarchy thus leading to one description of this architecture as being a "double-hierarchy". The online learning mechanisms can be regarded as a hierarchy because there is a tiered set of actions. At the highest level a population of solutions are stored in the Experience Bank and tested in a queue. If one of these stored experiences leads to a solution then the search ends, if none of these stored experiences leads to a solution then each of these experiences acquires fitness by the Experience Assessor depending how well each solution performed in the situation. The highest fitness experience is used as a starting position to the lower level GA that is used to generate new solutions to the current situation. This hierarchy preserves the system experience, and speeds up the genetic search by starting the genetic algorithm from the best found point in the space. This architecture had been used successfully to learn online and adapt robots behaviour in unstructured environments like agricultural robots and intelligent buildings. In their system the learning and adaptation techniques are inspired from Nature as in biology as most scientists agree that the remarkable adaptation of some complex organisms comes as a result of the interaction of two processes, working to different time scales: evolution and life long learning. Evolution takes place at the population level and determines the basic structures of the organism. Life long learning is responsible for some degree of adaptation at the individual level. It works by tuning the structures, built in accordance with the genetic information, by a process of gradual improvement of the adaptation to the surrounding environment [23]. They emulated the natural process by using evolution and online learning to develop a good enough controller of the robot and used their patented Fuzzy-Genetic system (the Associative Experience Engine) to speed the slow evolution process. An online adaptation technique is then used to implement the life long learning where the agent is always updating its knowledge and gaining experience and is able to adapt to the changing environment.

\subsection{Reinforcement Learning}

Reinforcement Learning (RL) is a learning strategy that can be applied to an agent that must learn its behaviour through trial and error interactions with a dynamic environment. RL is defined not by characterising a learning problem. Any algorithm that is well suited for solving the problem can be considered when using a reinforcement learning approach [9]. There are two main strategies for solving reinforcement learning problems. The first is to search the space of all possible behaviours in order to find one that performs well within the chosen environment. This is the approach normally taken by work involving genetic algorithms and genetic programming. The second is to use statistical techniques and dynamic programming methods to estimate the utility of taking particular actions in various world states [14]. This approach has been used to learn fuzzy systems for mobile robots in [1, 9, 27]. RL differs from the more widely studied problem of supervised learning in several ways. The most important difference is that there is no presentation of input/output pairs. Instead after choosing an action the agent is told the immediate reward and the subsequent state, but not told which action would have been in its best long-term interests. It is necessary for the agent to gather useful experience about the possible system states, actions, transitions and rewards actively to act optimally. 
Another difference from supervised learning is that on-line performance is important: the evaluation of the system is often concurrent with learning (which seems attractive in online learning in unstructured environments).

\section{This Special issue}

This special issue targets learning and adaptation in unstructured environments. The special issue introduces many interesting papers which we hope will enrich the field of learning and adaptation in unstructured environments. In this special issue many hard unstructured environments are presented such as underwater environments and mining environments and cluttered manipulators and piped robot environments. Also this special issue introduces novel learning and adaptation techniques in robotics operating in unstructured environments.

The paper entitled "2-Way Fuzzy Adaptive Identification and Control of a Flexible-Joint Robot Arm" by E. Gurkan, I. Erkmen, A. Erkmen presents their novel 2-way fuzy adaptive system that makes use of intuitionistic fuzzy sets to the identification and model based control of a flexible joint robot arm.

The paper entitled " Robot Learning with GA-Based Fuzzy Reinforcement Learning Agents" by Changjiu Zhou introduces a novel way to learn from both expert knowledge and measurement based information for a robot to acquire perception and motor skill. This paper introduces a general Genetic Algorithm based fuzzy reinforcement (GARFL) learning agent. Based on a neural fuzzy network architecture and how different kinds of expert knowledge and measurement based information can be incorporated in the GARFL agent so as to accelerate its learning. The GARFL was verified in the control of a piped robot

The paper entitled " A Behaviour Based Architecture for Autonomous Underwater Exploration" by J. Rosenblatt, S. Williams, H. Durant-Wayne, introduces a system for behaviour based control of an autonomous underwater vehicle for the purpose of inspection of coral reefs. Using sonar and vision based approaches, behaviours have been developed for guiding the robot along its intended course, for maintaining a constant height above the sea floor, and for avoiding obstacles. A behaviour arbitration has been implemented using both fuzzy logic and utility fusion.

The paper entitled "Knowledge Acquisition and Learning in Unstructured Robotic Assembly Environments" by I. Lopez, M. Howarth, introduces a creation of a self-adaptive robots based on the Adaptive Resonance Theory (ART) Artificial Neural Network (ANN). This paper shows how robots can operate autonomously in unstructured environments. This is achieved by providing the robot with a Primitive Knowledge Base (PKB) of the environment. This knowledge is gradually enhanced on-line based on the contact force information acquired during operations. The design of a novel Neural Network Controller (NNC) based on the Fuzzy ARTMAP network and its implementations results on an industrial robot are presented which validate the approach.

The paper entitled " Generation of Efficient Adjustment Strategies for a Fuzzy-Neuro Force Controller using Genetic Algorithms" by K. Kiguchi, K. Watanabe, T. Fukuda, presents an effective generation method of adjustment strategies for a fuzzy-neuro force controller (FNFC) of a robot manipulator in an unknown environment. In this method, strategies to adjust the FNFC in accordance with the environment dynamics are automatically generated in off-line manner using Genetic Algorithms. Fuzzy fitness evaluation method is proposed for the effective evolution of the neural network in the GA process. The effectiveness of the generated adjustment strategies of the FNFC has been evaluated by computer simulations with a 3DOF robot manipulator model.

The paper entitled " Reactive Navigation and Opportunistic Localisation for Autonomous Underground Mining Vehicles" by J. Roberts, E. Duff, P. Corke, describes an autonomous navigation system for a large underground mining vehicle. The control architecture is based on a robust reactive wall-following behaviour. The paper describes 
the implementation of a reactive navigation system on a 30 tonne Load-Haul-Dump truck. This truck has achieved full-speed autonomous operation at an artificial test mine, and subsequently at an operational underground mine.

The paper entitled " Sensor Based Learning for Practical Planning of Fine Motions in Robotics" by E. Cervera, A. Pobil, presents an implemented approach to part-mating of three dimensional non cylindrical parts with 6 DOF manipulator, considering uncertainties in modelling, sensing and control. The core of the proposed solution is a reinforcement learning algorithm for selecting the actions that achieve the goal in the minimum number of steps. Position and force sensor values are encoded in the state of the system by means of neural networks.

The paper entitled " Application of Online Neuro Fuzzy Controller to AUV", by T. Kim and J. Yuh, describes a neuro-fuzzy controller for autonomous underwater vehicles (AUVs) of which the dynamics are highly nonlinear, coupled, and time-varying. The neuro-fuzzy controller is based on the fuzzy membership function-based neural networks (FMFNN) with advantages of fuzzy logic and neural networks, such as inference capability and adoption of human operators' experience with fuzzy logic, and universal approximation and learning capability with neural networks. On-line learning of the FMFNN controller is achieved by using an inner-loop learning scheme and simplified derivatives of the vehicle system. Simulation results show effectiveness of the FMFNN controller for AUVs.

\section{References}

[1] H. Beom, H. Cho, A Sensor-Based Navigation for a Mobile Robot Using Fuzzy Logic and Reinforcement Learning, IEEE transactions on systems, man, cybernetics, Vol. 25, No. 3, (1995) 464-- 477.

[2] Bonarini, "Comparing Reinforcement Learning Algorithms Applied to Crisp and Fuzzy Learning Classifier systems", Proceedings of the Genetic and Evolutionary Computation Conference, (Orlando, Florida, July, 1999) 52-60.

[3] J. Borestein, Y. Koren, Real time obstacle avoidance for fast mobile robot, IEEE transactions on Systems, Man, Cybernetics, Vol. 19 (October 1989) 1179--1187.

[4] R. Brooks, Artificial Life and Real Robots, MIT press 1992.

[5] M. Delgado, F. Zuben, F. Gomide, Evolutionary design of Takagi-Sugeno Fuzzy Systems: a Modular and Hierarchical Approach, Proceedings of the 2000 IEEE International Conference on Fuzzy Systems, San AntonioUSA, May 2000.

[6] M. Dorigo, M. Colombetti, Robot Shaping: Developing Autonomous agents through learning, Artificial Intelligence Journal, Vol.71, (1995) 321--370.

[7] T. Fukuda, N. Kubota, "An Intelligent Robotic system based on Fuzzy approach", Proceedings of the IEEE, Vol. 87, No. 9, (September 1999) 1448--1470.

[8] Hagras, V. Callaghan, M. Colley, "Online Learning of Fuzzy Behaviours using Genetic Algorithms \& Real-Time Interaction with the Environment", Proceedings of the 1999 IEEE International Conference on Fuzzy Systems, (Seoul-Korea August 1999) 668-672.

[9] Hagras, V. Callaghan, M. Colley, "An Embedded-Agent Architecture for Online Learning and Control in Intelligent Machines", in New Frontiers in Computational Intelligence and its applications, (Eds. M. Mohammadin, IOS press 1999) 165--174.

[10]H. Hagras, V. Callaghan, M. Colley, Prototyping Design and Learning in Outdoor Mobile Robots operating in unstructured outdoor environments, IEEE International Magazine on Robotics and Automation, (September 2001) 53--69.

[11]H. Hagras, V. Callaghan, M. Colley, On Line Calibration of the sensors Fuzzy Membership Functions in Autonomous Mobile Robots, Proceedings of the 2000 IEEE International Conference on Robotics and Automation, (San Francisco-USA, April 2000) 3233--3238.

[12]H. Hagras, V. Callaghan, M. Colley, Learning Fuzzy Behaviour Co-ordination for Autonomous Multi-Agents Online using Genetic Algorithms \& Real-Time Interaction with the Environment, Proceedings of the 2000 IEEE International Conference on Fuzzy Systems, (San Antonio-USA, May 2000) 853--859.

[13]F. Hoffmann, Incremental Tuning of Fuzzy Controllers by means of Evolution Strategy, Proceedings of the GP98 Conference, (Madison, Wisconsin, 1998) 550--556.

[14] L. Kaelbing, M. Littman, Reinforcement Learning: A survey”, Journal of Artificial Intelligence Research, vol. 4 (1996) 237--285.

[15] N. Kasabov, Introduction: Hybrid intelligent adaptive systems. International Journal of Intelligent Systems, Vol.6, (1998) 453--454. 
[16]N. Kasabov, M. Watts, Neuro-Genetic Information Processing for Optimisation and Adaptation in Intelligent Systems, In: Neuro-Fuzzy Techniques for Intelligent Information Processing, N. Kasabov and R. Kozma, (Heidelberg Physica Verlag 1999) 97--110.

[17] O. Khatib, Real time obstacle avoidance for manipulators and mobile robots, International Journal of Robotics research, Vol.5, (1986) 90--98.

[18]D. Leitch, A New Genetic Algorithm for the Evolution of Fuzzy System, Ph.D. thesis, University of Oxford 1995.

[19] G. Linkens, O. Nyongeso, Genetic Algorithms for Fuzzy Control, Part II: Online System Development and Application, IEE proceedings Control theory applications, Vol.142 (1995) 177--185.

[20] Matellan, C. Fernandez, J. Molina, Genetic Learning for Fuzzy Reactive Controllers, Journal of Robotics and Autonomous Systems", Vol. 25, (1998) 33--41.

[21]O. Miglino, H. Lund, S. Nolfi, Evolving Mobile Robots in Simulated and Real Environments, Technical report NSAL-95007, Reparto di sistemi Neurali e vita Artificale, Instituto di Psicologia, Consiglio Nazionale delle Ricerche, Roma 1995.

[22] P. Pal, A. Kar, Mobile robot navigation using a neural network, Proceedings of the IEEE International Conference on Robotics and Automation, (1995) 1503--1508.

[23] M. Rocha, P. Cortez, J. Neves, The Relationship between Learning and Evolution in Static and Dynamic Environments, Proceedings of the Second ICSC Symposium on Engineering of Intelligent Systems, Paisley-UK, (June 2000) 500--506.

[24]L. Steels, When are Robots Intelligent Autonomous Agents", Journal of Robotics and Autonomous Systems, Vol. $15,(1995) 3--9$.

[25]E. Tunstel, T. Lippincott, M. Jamshidi, Behaviour Hierarchy for Autonomous Mobile Robots: Fuzzy Behaviour Modulation and Evolution", International Journal of Intelligent Automation and soft computing, Vol.3, No.1, (1997) 37--49.

[26] S. Vijayakumar, S. Schaal, Fast and Efficient incremental Learning for High-dimensional Movement Systems, Proceedings of the 2000 IEEE International Conference on robotics and Automation, (San Francisco-USA April 2000) 1894--1899.

[27] N. Yung, C. Ye, “ An Intelligent Mobile Vehicle navigator based on Fuzzy Logic and Reinforcement Learning", IEEE transactions on systems, man, cybernetics, Vol. 29, No. 2, (1999) 314--321. 